\title{
Prevalencia del síndrome de HELLP en gestantes críticas: Maternidad "Dr. Armando Castillo Plaza", Maracaibo, Venezuela
}

\author{
Laurenis Labarca. MSc ${ }^{1,2}$, José Ramón Urdaneta M. PhD 3 , María Elena González I. \\ $\mathrm{PhD}^{2,4}$, Alfi Contreras Benítez ${ }^{5}$, Nasser Saleh Baabel Z. PhD 4 , Mariem Fernández \\ Correa. ${ }^{1}$, Ángel Silva Bentacourt. ${ }^{1,2}$, María Lorena Nava. ${ }^{2}$
}

${ }^{1}$ Alumno Doctorado en Ciencias Médicas. Facultad de Medicina. Universidad del Zulia. ${ }^{2}$ Médico Adjunto Servicio Autónomo, Hospital Universitario de Maracaibo. 3Profesor Escuela de Bioanálisis, Facultad de Medicina, Universidad del Zulia. ${ }^{4}$ Profesor Escuela de Medicina, Facultad de Medicina, Universidad del Zulia. ${ }^{5}$ Profesor Cátedra de Anatomía. Facultad de Odontología. Universidad del Zulia. Venezuela.

\section{RESUMEN}

Objetivo: Determinar la prevalencia de síndrome de HELLP (SH) en gestantes críticamente enfermas ingresadas a la Unidad de Cuidados Intensivos Obstétricos (UCIO) de la Maternidad "Dr. Armando Castillo Plaza", de Maracaibo, estado Zulia, Venezuela, periodo 2011 a 2015. Métodos: Investigación descriptiva con diseño retrospectivo, donde se revisaron las historias clínicas de gestantes ingresadas a la UCIO con diagnóstico de $\mathrm{SH}$, analizándo sus características clínicas y epidemiológicas. Resultados: Hubo una prevalencia del $13,60 \%(111 / 816)$, tasa de letalidad de 1,23\% (10/816) y tasa de mortalidad materna específica de 15,08/100.000 nacidos vivos. Las características clínicas más prevalentes fueron: edad 25,8 $\pm 6,9$ años, estancia en UCIO 4,76 $\pm 4,46$ días, embarazos pretérminos $69,37 \%$, antecedentes de abortos $24,32 \%$, primíparas $42,34 \%$, control prenatal ausente o inadecuado, embarazos simples $95,5 \%$, preeclámpticas $67,57 \%$, antecedentes de condiciones preexistentes $47,75 \%$, sin hábitos como tabaco o alcohol $81,99 \%$, ingresaron embarazadas $89,19 \%$ y cesárea $74,77 \%$. Se diagnosticaron mayoritariamente casos de SH incompleto $(56,76 \%)$, siendo las complicaciones más observadas la disfunción hematológica $(98,2 \%)$, disfunción hepática $(91,9 \%)$ y disfunción renal $(70,3 \%)$. Conclusión: La prevalencia de SH resultó más elevada que lo reportada en estudios nacionales e internacionales, presentando características clínicas y epidemiológicas que deben ser consideradas para su prevención y diagnóstico precoz.

\section{PALABRAS CLAVE: Embarazo, embarazadas críticas, preeclampsia, síndrome de HELLP}

\section{SUMMARY}

Aims: To determinate the prevalence of HELLP syndrome (HS) in critical pregnant women admitted to the Obstetrics Intensive Care Unit (OICU) of the "Maternidad Dr. Armando Castillo Plaza", in Maracaibo, Zulia state, Venezuela, during 2011 to 2015. Methods: A descriptive research with retrospective design was due, in which were reviewed the clinical files of all pregnant admit into the OICU complicated with HS, was analyzed establish the clinical and epidemiological features. Results: We found a prevalence of $13.60 \%$ $(111 / 816)$, fatality rate of $1.23 \%(10 / 816)$ and specific maternal mortality rate of $15.08 / 100,000$ live births. The most prevalent clinical characteristics were: age $25.8 \pm 6.9$ years, stay in UCIO $4.76 \pm 4.46$ days, preterms pregnancies $(69.37 \%)$, history of abortions (24.32\%), primiparous $(42.34 \%)$, absent or inadequate prenatal care, singleton pregnancies $(95.50 \%)$, preeclampsia $(67.57 \%)$, history of pre-existing conditions $(47.75 \%)$, without habits such as tobacco or alcohol $(81.99 \%)$, admitted pregnant $(89.19 \%)$ and cesarean 
section $(74.77 \%)$. Also, were mainly diagnosed cases of incomplete HS $(56.76 \%)$; the most observed complications were hematologic dysfunction (98.2\%), liver dysfunction $(91.9 \%)$ and renal dysfunction $(70.3 \%)$. Conclusion: The prevalence of HELLP syndrome was higher than reported in national and international studies, presenting clinical and epidemiological characteristics that should be considered for prevention and early diagnosis.

\section{KEY WORDS: Pregnancy, critically ill pregnant, preeclampsia, HELLP syndrome}

\section{INTRODUCCIÓN}

La preeclampsia, con una incidencia entre 6-8\% de todos los embarazos, representa una de las causas más importantes de morbimortalidad materna en el mundo (1), atribuyéndose $15 \%-30 \%$ de todas las muertes maternas en México $(2,3,4), 12,3 \%$ en los Estados Unidos de América (1) y $29,59 \%$ en Venezuela para el año 2011 según estadísticas oficiales (5).

Dentro de las complicaciones de la preeclampsia, se encuentra el síndrome de HELLP, el cual es una de las complicaciones más temidas y significa por sus siglas en inglés H (Hemolysis), EL (Elevated Liver Enzimes), LP (Low Platelet Count) (6). El término de síndrome de HELLP fue propuesto por Weinstein en 1982, a pesar que sus alteraciones fueron descritas inicialmente por Pritchard en $1954(7,8)$, aunque no necesariamente se trata de un síndrome, se sigue utilizando como tal para dar continuidad a la descripción original (9).

Este síndrome puede desarrollarse en alrededor del $0,2-0,6 \%$ de todos los embarazos (10); principalmente en pacientes con preeclampsia severa y eclampsia, aunque puede diagnosticarse también en gestantes con hipertensión gestacional o preeclampsia agregada (11). A nivel mundial, la incidencia reportada en la literatura internacional, varía desde $2 \%$ hasta $12 \%$ en pacientes con preeclampsia-eclampsia (6); asimismo, es responsable de entre $3,5-10,5 \%$ de las muertes maternas y entre $7,4-20,4 \%$ de las muertes perinatales (12).

En Venezuela, algunos autores han revisado este entidad, reportándose una incidencia del 5,7\% en la Maternidad "Concepción Palacios" de Caracas (13); 4,9\% en Hospital "Dr. Adolfo Prince Lara" de Puerto Cabello, Estado Carabobo (14); 0,27\% en el Hospital "Joaquina de Rotondaro" de TinaquiIlo, Estado Cojedes, y las más recientes de la Maternidad "Concepción Palacios" que reportan una incidencia del $38,23 \%$ en eclámpticas (15) y otra que registra una incidencia durante el embarazo de $0,13 \%$ y en preeclampsia-eclampsia del $3,29 \%$ (6). Por su parte, en el estado Zulia se reportó para el periodo 2004 - 2009 entre todos los casos que ingresaron a la unidad de cuidados intensivos
(UCI) del Hospital Universitario de Maracaibo una incidencia del $1,8 \%$ con $29 \%$ de muertes maternas (16).

Aunque su fisiopatología es desconocida, el síndrome HELLP es la traducción de la diversidad biológica de microangiopatías maternas que puede ocurrir durante la preeclampsia y se han reportado múltiples alteraciones bioquímicas y genéticas en las pacientes estudiadas (17). Su historia natural ha cambiado favorablemente, al menos desde los últimos veinte años, debido a que las enfermas que lo padecen reciben tratamiento multidisciplinario más agresivo, cada vez más practicado en unidades de cuidados críticos obstétricos (UCIO), y con medidas intervencionistas que incluyen el uso de corticoesteroides del tipo dexametasona, la interrupción gestacional y atención del parto de manera oportuna y segura; asimismo, dado el creciente avance en el diagnóstico y terapéutica de la enfermedad y con base en la experiencia de cada centro de atención terciaria, se espera que la frecuencia y tipo de complicaciones perinatales se modifique al paso de los años (9).

Con base a estos planteamientos, se propuso determinar la prevalencia del Síndrome de HELLP en gestantes ingresadas a la UCIO de la Maternidad "Dr. Armando Castillo Plaza", de Maracaibo, estado Zulia, durante el periodo 2011-2015.

\section{PACIENTES Y MÉTODOS}

Se realizó una investigación descriptiva con diseño, retrospectivo, de prevalencia y longitudinal, realizado en la UCIO de la Maternidad "Dr. Armando Castillo Plaza" de la ciudad de Maracaibo, estado Zulia, Venezuela, durante el periodo comprendido entre los años 2011 y 2015. Se tomó un censo poblacional, incluyéndose todas las pacientes que ingresaron a esta unidad con dicho diagnóstico, para lo cual se revisaron todas las historias clínicas de pacientes que ingresaron a la $\mathrm{UCIO}$ registradas bajo el código O14.2 correspondiente a este síndrome según la $10^{\underline{a}}$ revisión del Código Internacional de Enfermedades y Problemas de Salud Conexos (CIE10) de la OMS (18). Asimismo, para evitar sesgos y el sub-registro se revisaron todas aquellas historias 
codificadas como $\mathrm{O} 10$ - O16 (Edema, proteinuria y trastornos hipertensivos en el embarazo, parto y puerperio).

Se procedió a asentar en una ficha de trabajo las características generales de las pacientes: (a) edad, (b) tiempo transcurrido desde el ingreso en la institución y su traslado a la $\mathrm{UCl}$, definido como menos de 6 horas, de 6 a 24 horas y más de 24 horas; y (c) estado de la paciente al ingreso (embarazada o puérpera). Seguidamente se analizaron las siguientes cualidades:

(a) Morbilidad: se establecieron los diagnósticos de ingreso de las pacientes a la institución.

(b) Factores de riesgos: tales como la procedencia (rural o urbana), estado civil (soltera, casada, divorciada o viuda), escolaridad (último nivel académico alcanzado), control prenatal (adecuado si al momento del evento la paciente había asistido a por lo menos 3 consultas de atención prenatal), paridad (primiparidad o multiparidad), edad materna (precoz menor a 19 años o avanzada mayor a 35 años), edad gestacional (pretérmino menor a 37 semanas, a término entre 37 y 42 semanas y postérmino mayor a 42 semanas), tipo de embarazo (simple o gemelar), tipo de parto (eutócico o distócico), hábitos (alcohol, tabaco, drogas ilícitas), antecedentes mórbidos (enfermedad propia de la gestación o condición médica preexistente definida como cualquier enfermedad o síndrome, actual o pasado, que conste en la historia clínica o refiera la paciente o su familia al ingreso, por la que pudo haberse estudiado, recibido o no tratamiento ambulatorio o en internación, incluyendo tratamientos quirúrgicos).

(c) Estancia hospitalaria: número de días de estadía en la UCI.

(d) Complicaciones, agrupadas por sistemas: Disfunción cardiovascular (hipotensión debida a hipovolemia, vasodilatación periférica con descenso de los índices de resistencia e impacto en la función ventricular izquierda), Disfunción respiratoria (descenso en la $\mathrm{PaO} 2$, taquipnea y signos de edema pulmonar), Disfunción gastrointestinal (disminución en la perfusión de la mucosa intestinal, intolerancia al soporte nutricional, úlceras de estrés, pancreatitis y disfunción hepática), Disfunción renal (disminución en el flujo sanguíneo renal con oliguria y elevación de azoados), Disfunción hematológica (cambios en el recuento de glóbulos blancos por leucopenia o leucocitosis severa, anormalidades en la coagulación y coagulación intravascular diseminada), Disfunción del sistema nervioso central (alteración del estado de conciencia y coma), o falla orgánica múltiple (disfunción de 2 o más órganos o sistemas). (e) Tratamiento: intervenciones realizadas, definidas como soporte inotrópico, ventilatorio, transfusiones de hemoderivados, hipotensores (sulfato de magnesio $u$ otros antihipertensivos), sedación-analgesia, cirugía (histerectomía u otras intervenciones quirúrgicas).

(f) Tasa de mortalidad materna: definida como el número de muerte de una mujer durante el embarazo, parto o puerperio, calculadas de manera global.

Los datos obtenidos se analizaron mediante el Paquete Estadístico para Ciencias Sociales (SPSS), versión 19, ejecutándose un tratamiento estadístico descriptivo, donde los resultados obtenidos se expresaron mediante frecuencias absolutas y relativas (porcentajes), medidas de tendencia central (medias) y de dispersión (desviación estándar); estos resultados se muestran en cuadros de distribución de frecuencias.

\section{RESULTADOS}

Durante el quinquenio 2011-2015 fueron admitidas a la UCIO de la Maternidad "Dr. Armando Castillo Plaza" 111 pacientes con diagnóstico presuntivo de síndrome HELLP, lo cual equivale a una prevalencia de 13,60\% (Tabla I). Se evidenció que existe una tendencia irregular a través de los años, en cuanto a la morbilidad proporcional, observándose la mayor tasa para los años 2011 (24,11\%) y $2015(18,54)$; en tanto que las tasas más bajas se presentaron en los años 2013 y 2014 (6,93\% y $6,96 \%$ respectivamente). En cuanto a la mortalidad, la tasa de letalidad por síndrome HELLP entre todas las causas de ingreso a la UCIO fue de $1,23 \%(10 / 816)$ y al medir el riesgo de muerte por esta causa, mediante la tasa de mortalidad materna específica, esta fue de 15,08/100.000 nacidos vivos; evidenciándose fluctuaciones con tendencias ascendentes y presentándose las mayores tasas en los años 2014 y 2015 (26,8 y 24,27/100.000 nacidos vivos, respectivamente).

Asimismo, se encontró que la edad promedio de las pacientes ingresadas era de 25,8 \pm 6,9 años, quienes presentaron una estancia promedio en UCIO de 4,76 \pm 4,46 días (rango: 1-23 días) y de estancia en sala de hospitalización de 9,32 \pm 5,89 días (rango: 1-35 días). En cuanto a las características demográficas de las pacientes ingresadas (Tabla II) se evidenció que destacan el predominio de la edad adecuada para la gestación $(63,06 \%)$, raza indígena $(58,56 \%)$, procedencia urbana $(52,25 \%)$, estado civil concubina $(58,56 \%)$ y escolaridad secundaria $(60,36 \%)$. 
Tabla I

MORBIMORTALIDAD POR SÍNDROME DE HELLP. UCIO-MATERNIDAD “DR. ARMANDO CASTILLO PLAZA”, 2011- 2015

\begin{tabular}{lcccccc}
\hline Año & $\begin{array}{c}\text { Ingreso por } \\
\text { SH }\end{array}$ & $\begin{array}{c}\text { Ingreso todas } \\
\text { las causas }\end{array}$ & $\begin{array}{c}\text { Morbilidad proporcional } \\
\text { por SH * }\end{array}$ & $\begin{array}{c}\text { Muertes Maternas } \\
\text { por SH }\end{array}$ & $\begin{array}{c}\text { Nacidos } \\
\text { vivos }\end{array}$ & $\begin{array}{c}\text { TMM } \\
\text { por SH }\end{array}$ \\
\hline 2011 & 34 & 141 & 24,11 & 0 & 10875 & 0,00 \\
2012 & 23 & 150 & 15,33 & 1 & 16117 & 6,20 \\
2013 & 12 & 173 & 06,93 & 3 & 16135 & 18,58 \\
2014 & 14 & 201 & 06,96 & 4 & 14909 & 26,80 \\
2015 & 28 & 151 & 18,54 & 2 & 8240 & 24,27 \\
\hline Total & 111 & 816 & 13,60 & 10 & 66276 & 15,08 \\
\hline
\end{tabular}

* $100 \%$ corresponden a los ingresos a UCl en cada año. ** Tasa de mortalidad materna por 100.000 Nacidos vivos. SH: Síndrome de HELLP.

\section{Tabla II \\ CARACTERIZACIÓN DEMOGRÁFICA DEL SÍNDROME DE HELLP. UCIO-MATERNIDAD “DR. ARMANDO CASTILLO PLAZA", 2011- 2015}

\begin{tabular}{|c|c|c|}
\hline Características & $\mathrm{n}$ & $\%$ \\
\hline \multicolumn{3}{|l|}{ Edad (años): } \\
\hline Precoz $(\leq 19)$ & 26 & 23,42 \\
\hline Adecuada(20-35) & 70 & 63,06 \\
\hline Avanzada (>35) & 15 & 13,52 \\
\hline \multicolumn{3}{|l|}{ Raza: } \\
\hline Blancas & 37 & 33,33 \\
\hline Indígenas & 65 & 58,56 \\
\hline Afrodescendientes & 9 & 8,11 \\
\hline \multicolumn{3}{|l|}{ Procedencia: } \\
\hline Urbana & 58 & 52,25 \\
\hline Rural & 53 & 47,75 \\
\hline \multicolumn{3}{|l|}{ Estado civil: } \\
\hline Concubina & 65 & 58,56 \\
\hline Soltera & 37 & 33,33 \\
\hline Casada & 08 & 7,20 \\
\hline Viuda & 01 & 0,90 \\
\hline \multicolumn{3}{|l|}{ Escolaridad: } \\
\hline Primaria & 38 & 34,23 \\
\hline Secundaria & 67 & 60,36 \\
\hline Técnica & 6 & 5,41 \\
\hline
\end{tabular}

Respecto a las características clínicas (Tabla III), destacaron embarazos pre-términos $(69,37 \%)$, antecedentes de abortos previos (24,32\%), primiparidad $(42,34 \%)$, control prenatal ausente 0 inadecuado $(63,96 \%)$, condición de la madre al ingreso como embarazada $(89,19 \%)$, embarazos simples $(95,50 \%)$, tipo de trastornos hipertensivo: preeclampsia $(67,57 \%)$, cesárea $(74,77 \%)$, pacientes sin hábitos como tacaco o alcohol $(81,99 \%)$ y pacientes con condiciones preexistentes $(47,75 \%)$. Con relación a la clasificación del síndrome (Tabla IV), predominó el tipo incompleto en un $56,76 \%$; mientras que los factores de riesgo específicos para el síndrome de HELLP (Tabla V) observados con mayor frecuencia fueron la edad mayor de 25 años $(50,45 \%)$ y la multiparidad $(34,23 \%)$.

Por otra parte, entre los signos y síntomas más frecuentes observados en estas pacientes (Tabla $\mathrm{VI}$ ), ocupando los primeros lugares en igual porcentaje se encontró la hipertensión e hiperreflexia $(91,89 \%)$, seguido de cefalea $(59,46 \%)$ y edema $(23,42 \%)$; asimismo, se registraron la presión arterial diastólica, presión arterial sistólica, presión arterial media y presión venosa central, las cuales tuvieron un promedio de $104,96 \pm 10,7 \mathrm{mmHg}$, $158,26 \pm 15,8 \mathrm{mmHg}, 122,31 \pm 11,6 \mathrm{mmHg}$ y 10,23 $\pm 2,5 \mathrm{mmH} 2 \mathrm{O}$, respectivamente. Respecto a las complicaciones observadas (Tabla VII) destacaron la disfunción hematológica $(98,2 \%)$, disfunción hepática $(91,9 \%)$ y disfunción renal $(70,3 \%)$ como las más observadas. 
Tabla III

CARACTERIZACIÓN CLÍNICA DEL SÍNDROME DE HELLP.

UCIO-MATERNIDAD “DR. ARMANDO CASTILLO PLAZA", 2011- 2015

\begin{tabular}{|c|c|c|}
\hline Características & $\mathrm{n}$ & $\%$ \\
\hline \multicolumn{3}{|l|}{ Edad gestacional: } \\
\hline Pretérmino & 77 & 69,37 \\
\hline Término & 34 & 30,63 \\
\hline \multicolumn{3}{|l|}{ Antecedentes obstétricos: } \\
\hline Antecedentes de abortos & 24 & 21,62 \\
\hline Cesáreas previas & 16 & 14,41 \\
\hline \multicolumn{3}{|l|}{ Paridad: } \\
\hline Primiparidad & 47 & 42,34 \\
\hline II-IV paras & 26 & 23,42 \\
\hline Multiparidad (>5) & 38 & 34,23 \\
\hline \multicolumn{3}{|l|}{ Control prenatal: } \\
\hline Ausente & 30 & 27,02 \\
\hline Inadecuado & 41 & 36,94 \\
\hline Adecuado & 40 & 36,04 \\
\hline \multicolumn{3}{|l|}{ Condición de la madre al ingreso: } \\
\hline Embarazada & 99 & 89,19 \\
\hline Puérpera & 12 & 10,81 \\
\hline \multicolumn{3}{|l|}{ Tipo de trastorno hipertensivo: } \\
\hline Preeclampsia & 75 & 67,57 \\
\hline Eclampsia & 10 & 9,01 \\
\hline HTA Crónica + Preeclampsia Agregada & 26 & 23,42 \\
\hline \multicolumn{3}{|l|}{ Tipo de embarazo: } \\
\hline Simple & 106 & 95,50 \\
\hline Gemelar & 5 & 4,50 \\
\hline \multicolumn{3}{|l|}{ Vía de parto: } \\
\hline Cesárea & 83 & 74,77 \\
\hline Vaginal & 28 & 25,23 \\
\hline \multicolumn{3}{|l|}{ Hábitos: } \\
\hline Sin hábitos & 91 & 81,99 \\
\hline Tabaco & 15 & 13,51 \\
\hline Alcohol & 5 & 4,50 \\
\hline \multicolumn{3}{|l|}{ Antecedentes mórbidos: } \\
\hline Sin antecedentes & 51 & 45,95 \\
\hline Condición preexistente & 53 & 47,75 \\
\hline Enfermedad de la gestación & 7 & 6,30 \\
\hline
\end{tabular}

Tabla IV

CLASIFICACIÓN DEL SÍNDROME DE HELLP. UCIO-MATERNIDAD “DR. ARMANDO CASTILLO PLAZA", 2011- 2015

\begin{tabular}{lcc}
\hline Clasificación & $\mathrm{n}$ & $\%$ \\
\hline Completo & 48 & 43,24 \\
Incompleto & 63 & 56,76
\end{tabular}

Tabla V

FACTORES DE RIESGO ESPECÍFICO PARA SÍNDROME DE HELLP. UCIO-MATERNIDAD "DR. ARMANDO CASTILLO PLAZA", 2011- 2015

\begin{tabular}{lcc}
\hline Factor de riesgo & $\mathrm{n}$ & $\%$ \\
\hline Multiparidad & 38 & 34,23 \\
Edad mayor de 25 años & 56 & 50,45 \\
Raza blanca & 37 & 33,33 \\
Antecedentes de abortos & 24 & 21,62 \\
\hline
\end{tabular}

\section{Tabla VI \\ SÍNTOMASY SIGNOS EN PACIENTES CON SÍNDROME DE HELLP. UCIO-MATERNIDAD “DR. ARMANDO CASTILLO PLAZA", 2011- 2015}

\begin{tabular}{lcc}
\hline Manifestaciones clínicas & $\mathrm{n}$ & $\%$ \\
\hline Epigastralgia & 24 & 21,62 \\
Cefalea & 66 & 59,46 \\
Edema & 26 & 23,42 \\
Alteraciones visuales & 20 & 18,02 \\
Hipertensión arterial & 102 & 91,89 \\
Hiperreflexia & 102 & 91,89 \\
Sangrado genital & 16 & 14,41 \\
Estupor & 08 & 7,21 \\
Movimientos tónicos-clónicos & 12 & 10,81 \\
\hline
\end{tabular}




\section{Tabla VII \\ COMPLICACIONES EN PACIENTES CON SÍN- DROME DE HELLP. \\ UCIO-MATERNIDAD “DR. ARMANDO CASTILLO PLAZA", 2011- 2015}

\begin{tabular}{lcc}
\hline Complicaciones & $\mathrm{n}$ & $\%$ \\
\hline Disfunción cardiovascular & 28 & 25,22 \\
Disfunción respiratoria & 14 & 12,61 \\
Disfunción renal & 78 & 70,27 \\
Disfunción hematológica & 109 & 98,19 \\
Falla orgánica múltiple & 7 & 6,30 \\
Insuficiencia renal aguda & 21 & 18,92 \\
Disfunción hepática & 102 & 91,89 \\
Eclampsia & 22 & 19,82 \\
Desprendimiento prematuro de placenta & 2 & 1,80 \\
Derrame pleural & 7 & 6,30 \\
Ascitis severa & 5 & 4,50 \\
Edema cerebral & 2 & 1,80 \\
Falla hepática & 5 & 4,50 \\
Infección herida quirúrgica & 3 & 2,70 \\
Sepsis & 12 & 10,81 \\
\hline
\end{tabular}

Finalmente, en lo concerniente a las pruebas de laboratorio realizadas al ingreso de las pacientes a la UCIO (Tabla VIII), se evidenciaron alteraciones hematológicas con tendencia a la disminución en los valores de hemoglobina, cuya media oscila entre un mínimo de $3,5 \mathrm{~g} / \mathrm{dl}$ y un máximo de $12,3 \mathrm{~g} / \mathrm{dl}$ y un promedio de $8,33 \pm 1,72 \mathrm{~g} / \mathrm{dl}$; en concordancia con estos resultados, el hematocrito se encuentra disminuido con un promedio de 27,50 $\pm 5,74$ (rango: $12 \%-39 \%$ ). Igualmente, los valores de plaquetas se encontraron por debajo de los valores referenciales normales $\left(90.184,6 \pm 29.098,33 / \mathrm{mm}^{3}\right)$.

De igual manera, entre las pruebas del perfil hepático con valores superiores a los valores referenciales se encontraron las transaminasas TGO y TGO en $99,12 \%$ de las pacientes; al igual que la bilirrubina total $(71,2 \%)$, directa $(58,6 \%)$ e indirecta $(84,7 \%)$ y la lactato deshidrogenasa DHL $(96,4 \%)$. Por otra parte, entre las pruebas del perfil renal se encontraron principalmente afectada la urea con valores aumentados de la muestra $(51,42 \%)$, al igual que las concentraciones séricas de ácido úrico $(88,35 \%)$ y las proteínas totales $(79,36 \%)$; en tanto que en los valores de creatinina predominaron normales en $70,31 \%$ y los niveles séricos de albumina disminuidos en $79,38 \%$ de los casos.

\section{Tabla VIII \\ PARÁMETROS DE LABORATORIO EN PACIENTES CON SÍNDROME DE HELLP. UCIO-MATERNIDAD "DR. ARMANDO CASTILLO PLAZA", 2011- 2015}

\begin{tabular}{|c|c|c|}
\hline Parámetros & $\bar{x}$ & $\pm \mathrm{DE}$ \\
\hline Hemoglobina (g/dl) & 8,33 & 1,72 \\
\hline Hematocrito (\%) & 27,50 & 5,74 \\
\hline Plaquetas $\left(\mathrm{x} \mathrm{mm}^{3}\right)$ & $90.184,6$ & $29.098,33$ \\
\hline Urea (mg/dl) & 49,62 & 49,68 \\
\hline Creatinina (mg/dl) & 1,45 & 1,44 \\
\hline Albumina (g/dl) & 2,90 & 0,71 \\
\hline Proteínas totales (g/dl) & 5,41 & 1,35 \\
\hline $\mathrm{LDH}(\mathrm{U} / \mathrm{L})$ & $1.156,50$ & 874,02 \\
\hline Ácido úrico (mg/dl) & 7,44 & 1,63 \\
\hline TGO (U/L) & 256,60 & 244,04 \\
\hline TGP (U/L) & 236,62 & 264,20 \\
\hline Bilirrubina total (mg/dl) & 2,03 & 2,51 \\
\hline Bilirrubina directa (mg/dl) & 0,85 & 1,36 \\
\hline Bilirrubina indirecta $(\mathrm{mg} / \mathrm{dl})$ & 1,18 & 1,27 \\
\hline
\end{tabular}

\section{DISCUSIÓN}

A nivel mundial, la prevalencia reportada en la literatura internacional, varía desde $2 \%$ hasta $12 \%$ (6), un poco más elevado que lo encontrado en este estudio que demuestran una prevalencia de $13,60 \%$; pero más o menos similar al $11,44 \%$ publicado en México por Vásquez y Flores (9). Al comparar este resultado con otras investigaciones efectuadas en el ámbito nacional, esta prevalencia resulta ser mucho menor que el $62,5 \%$ encontrado en la Maternidad Concepción Palacios de la ciudad de Caracas (6), pero mucho mayor al 0,27\% reportado en Tinaquillo, estado Cojedes (19), al 5,7\% en Caracas (13), 4,9\% en Hospital "Dr. Adolfo Prince Lara" de Puerto Cabello, Estado Carabobo (14) y al $0,12 \%$ reportado en una $\mathrm{UCl}$ general de Maracaibo por Pavajeau (16). Este amplio rango de variación se podría explicar, entre otras causas, por los distintos criterios utilizados para definir el diagnóstico de esta enfermedad (6).

A diferencia de otro estudios realizados tanto en México (9) como en Venezuela $(6,19)$ donde no se registraron muertes debido a este síndrome, este estudio estableció una tasa específica de mortalidad materna de 15,08/100.000 nacidos vivos; lo 
cual contrapone a la elevada mortalidad (29\%) presentada en otra investigación realizada en la UCI de adultos de esta misma institución (16), antes de la apertura de la UCIO. En este contexto, se ha establecido que mundialmente alrededor del 3,5\% al $10,5 \%$ de casos de muertes maternas se producen a consecuencia de este síndrome (12), e inclusive pudiendo alcanzar hasta un $24 \%$ (20).

Con relación a la edad de las pacientes ingresadas en esta UCIO tenían una edad promedio de 25 años, similar a la edad de las pacientes de otros estudios realizados tanto en Venezuela (19) como en la Argentina (12); pero mucho menor a la edad promedio reportada en otra investigación donde resultó en promedio de $30 \pm 6,91$ años (9). También la edad de estas pacientes resultó mayor que la publicada por otro estudio realizado en la localidad, donde la mayor parte de los ingresos (31\%) se presentó en adolescentes (16).

Respecto a las características clínicas de las pacientes ingresadas por esta causa, al igual que lo reportado en un estudio efectuado en la capital venezolana por Soto y cols (6), este síndrome se presentó principalmente en primigestas y en embarazos pretérminos, a diferencia de otro estudio venezolano que encontró que la mayoría de los casos se identificaron en embarazos a término (19). Asimismo, se encontró que a la mayor parte de estas pacientes $(74,77 \%)$ les fue practicada la operación cesárea como vía de parto, similar a lo publicado por investigadores mexicanos con un $91,67 \%$ (9).

En lo que concierne al diagnóstico de esta patología, en la mayoría de los casos se realizó durante el embarazo $(89,19 \%)$, al igual que otro estudio nacional (19) donde principalmente se diagnosticó antes del parto $(68,42 \%)$; difiriendo de lo reportado en Argentina por Vargas y cols (12), donde la mayoría de los casos $(59,7 \%)$ les fue diagnosticada la patología en el puerperio inmediato. Vale destacar que se han reportado casos atípicos donde ocurre una exacerbación postparto e inclusive inicio antes de las 20 semanas de embarazo, con ausencia de proteinuria o hipertensión (10).

Por otro lado, en esta serie predominó el diagnóstico, de acuerdo a la clasificación de Sibai y cols (21), del síndrome de HELLP incompleto (56,76\%), a diferencia de los resultados presentados por Aydin y cols (22), donde las formas completas e incompletas de este síndrome fueron más o menos similares (64/131 vs. 67/131). En cuanto a los factores de riesgo relacionados con mayor incidencia del síndrome de HELLP destacan: la multiparidad, edad materna mayor a 25 años, raza blanca y el antecedente de abortos (23).

En relación a la sintomatología clínica, esta investigación determinó un predominio de la elevación de las cifras de presión arterial e hiperreflexia, lo cual difiere de lo encontrado por García y cols (8) en Chile, donde el dolor en epigastrio resultó ser el hallazgo clínico que mayormente sugería tempranamente el desarrollo del síndrome de HELLP, mientras que la hipertensión puede estar ausente hasta en un $20 \%$ de las pacientes.

Las pacientes con este síndrome pueden presentar complicaciones maternas graves, las cuales se describen como intrahepáticas o multisistémicas, y pueden sobrevenir durante el embarazo o el puerperio y en solitario o combinadas (9). En estas pacientes prevalecieron como complicaciones las disfunciones de tipo hematológica, hepática y renal; mientras que en otros estudios realizados en el país la principal complicación resultaron ser la insuficiencia renal aguda (6), las complicaciones úteroplacentarias (19) y complicaciones respiratorias asociadas a ventilación mecánica prolongada (16). Al respecto, una investigación mexicana (9) determinó que la frecuencia de las complicaciones maternas resultó muy baja, presentándose principalmente ascitis $(21,06 \%)$ y abrupto placentario $(10,53 \%)$.

Con relación a las pruebas de laboratorio realizadas al ingreso a la UCIO, se evidenció en casi la totalidad de las pacientes una elevación de las transaminasas hepáticas; al compararse con los hallazgos presentados por una investigación previa realizada en la UCI general de esta institución (16), los valores promedios de estas enzimas al ingreso de las pacientes fueron casi cinco veces más elevados. Al respecto, se ha reportado que las transaminasas elevadas sugieren tempranamente el desarrollo de síndrome de HELLP, pues estas se encontraban significativamente mayores en las pacientes con preeclampsia severa que desarrollan síndrome de HELLP, observándose que son las primeras pruebas bioquímicas en alterarse (8).

Otros estudios han determinado que el síndrome de HELLP se asocia a una alta tasa de morbimortalidad perinatal (74-200/1000 nacidos vivos), asociada principalmente a complicaciones de prematuridad $(6,8,12,24,25)$; por tanto, una limitación del presente estudio es que no pudo dársele seguimiento a los productos y se imposibilitó el acceso a sus respectivos expedientes clínicos puesto que éstos son codificados con un número diferente y no se asocian a las historias clínicas de las madres. Sin embargo una fortaleza de este estudio, es que al compararlo con una investigación previa efectuada en esta misma institución antes disponer de una $\mathrm{UCI}$ exclusiva para la atención de pacientes obstétricas, se pone en evidencia que si bien hay una clara tendencia al aumento de la prevalencia de estos casos, la mortalidad por esta causa ha disminuido notablemente.

En este orden de ideas, la presente investigación denota la importancia de que en cada centro de atención materna se disponga de un equipo multidisciplinario de especialistas en medicina materno-fetal y en cuidado crítico obstétricos, para el 
difícil entendimiento y manejo de la historia natural de las enfermedades y sus complicaciones durante la gestación, el parto y el puerperio, de manera de poder tomar decisiones acertadas y oportunas para el tratamiento de las pacientes obstétricas críticas, a fín de reduccir la mortalidad materna.

\section{CONCLUSIÓN}

La prevalencia de síndrome de HELLP resultó más elevada que lo reportada en estudios nacionales e internacionales, presentando características clínicas y epidemiológicas que deben ser consideradas para su prevención y diagnóstico precoz. Tal como lo demuestran los resultados presentados es imprescindible que se mejoren los programas de atención prenatal y se aumente su cobertura, de modo que puedan detectarse precozmente los trastornos hipertensivos del embarazo y evitar la presencia de algunas de sus complicaciones, como lo es el síndrome de HELLP.

\section{REFERENCIAS}

1. Moser M, Brown CM, Rose CH, Garovic VD. Hypertension in pregnancy: is it time for a new approach to treatment? J Hypertens 2012;30(6):1092-100.

2. Villanueva LA, Collado SP. Conceptos actuales sobre la preeclampsia-eclampsia. Rev Fac Med UNAM 2007;50(2):57-61.

3. Castelazo E, Monzalbo DE, López M J, Castelazo S. Preeclampsia atípica y éxito perinatal: reporte de caso. Ginecol Obstet Mex 2014;82(1):70-4.

4. Romero G, Hernández BO. Persistencia de hipertensión en mujeres con preeclampsia. Ginecol Obstet Mex 2011;79(10):601-6.

5. Ministerio del Poder Popular para la Salud. (2011). Anuario de Mortalidad 2011. [Documento en línea]. Disponible en: http://www.mpps.gob.ve/modules.php ?name=Downloads\&op=search\&query. Acceso: 1911-2014.

6. Soto F, Rivera L, Estévez M, Ayala V, Cabrera C. Síndrome HELLP: morbilidad-mortalidad materna y perinatal. Rev Obstet Ginecol Venez 2014;74(4):244-51.

7. Haram K, Svendsen E, Abildgaard U. The HELLP syndrome: Clinical issues and management. A review. BMC Pregnancy Childbirth 2009;9:8. [Periódico en línea]. Disponible en: http://bmcpregnancychildbirth. biomedcentral.com/articles/10.1186/1471-2393-9-8. Acceso: 08-01-2015.

8. García V, Echavarría LG, Ardila R, Gutiérrez J. Hallazgos clínicos y de laboratorio que sugieren tempranamente el síndrome de HELLP en pacientes con preeclampsia severa. Rev Chil Obstet Ginecol 2014;79(1):9-13.

9. Vázquez JG, Flores CX. Complicaciones maternas en pacientes con síndrome de HELLP. Ginecol Obstet Mex 2011;79(4):183-9.

10. Garrido MF, Carvajal JA. Síndrome de HELLP normotensivo: caso clínico. Rev Med Chile 2013;141(11):1470-4.
11. Vasco M, Vigil-De Gracia P. Abordaje del anestesiólogo en pacientes con hipertensión y embarazo. Rev Obstet Ginecol Venez 2014;74(1):15-23.

12. Vargas C, Aguilera R, Romero G, Andrade JY, Bustillo MC. Prevalencia del Síndrome de HELLP y sus resultados Perinatales en pacientes del Hospital Materno Infantil. Rev Med Post UNAH. 2010; 13 (3) [Periódico en línea]. Disponible en: http://www.lamjol.info/index. php/RMPM/article/view/630. Acceso: 15-12-14.

13. Fleitas F, Serfati M, Garcia M, Weibezahn H, Llovera A, Machado A. Síndrome HELLP: Revisión de los años 1991-1993. Rev Obstet Ginecol Venez 1997;57(4):229-35.

14. López JR, Rivas M, Colmenares B, Alvarado S, Silva D, Capretta D. Síndrome HELLP en la Maternidad del Hospital "Dr. Adolfo Prince Lara". Rev Obstet Ginecol Venez 2001;61(2):77-81.

15. Valarino G, Mora A, Cabrera C, Durán I, Díaz Y, González S, et al. Eclampsia. Morbilidad y mortalidad materna y perinatal. Rev Obstet Ginecol Venez 2009;69(3):152-61.

16. Pavajeau JD. Características epidemiológicas de pacientes con Síndrome de HELLP ingresados a la unidad de cuidados intensivos del Hospital Universitario de Maracaibo. Universidad del Zulia. Facultad de Medicina. Programa de Especialización en Medicina Crítica. 2009. [Tesis en línea]. Disponible en: http://tesis.luz.edu.ve/tde_arquivos/20/TDE-2011-1027T13:41:46Z 1991/Publico/pavajeau_ospino_jesus_ dario.pdf. Acceso: 5-11-14.

17. Rivas EE, Mendivil C. Síndrome de Hellp: Revisión. Salud Uninorte 2011;27(2):259-74.

18. World Health Organization. Statistical Classification of Diseases and Related Health Problems (ICD): 10th rev. Geneva, Switzerland. 2007. [Documento en línea] Disponible en: http://apps.who.int/classifications/ apps/icd/icd10online/. Acceso: 04-01-2015

19. Suárez $R$, Suárez $R$, Suárez $F$, Tobon $D$, Agüin $V$. Síndrome HELLP en la Maternidad del Hospital "Joaquina de Rotondaro", Tinaquillo, estado Cojedes, 2008-2009. Acta Científica Estudiantil 2009;7(2):1006.

20. Vigil-De Gracia P. Maternal deaths due to eclampsia and HELLP syndrome. Int J Gynecol Obstet 2009;104(2):90-4.

21. Sibai BM. The HELLP syndrome (hemolysis, elevated liver enzymes; and low platelet): Much ado about nothing? Am J Obstet Gynecol 1990;162(2):311-6.

22. Aydin S, Ersan F, Ark C, Arioğlu Aydin C. Partial HELLP syndrome: maternal, perinatal, subsequent pregnancy and long-term maternal outcomes. J Obstet Gynaecol Res 2014;40(4):932-40.

23. Gutiérrez CH, Alatorre J, Cantú O, Gómez D. Síndrome de HELLP, diagnóstico y tratamiento. Rev Hematol Mex 2014;13(4):195-200.

24. Joshi D, James A, Quaglia A, Westbrook RH, Heneghan MA. Liver disease in pregnancy. Lancet 2010;375(9741):594-605.

25. Guzel AL, Kuyumcuoglu U, Celik Y. Are maternal and fetal parameters related to perinatal mortality in HELLP syndrome? Arch Gynecol Obstet 2011;283(6):122732. 